\title{
Modelado y simulación de conversión de energía eólica PMSG para sistema aislado
}

Rubén Domingo Bufanio ${ }^{1}$

\section{Resumen}

Existe hoy en día, dado aspectos técnico económicos, factibilidad de suministrar energía eléctrica a través de generación eólica, en donde el recurso lo permita, a pequeñas comunidades aisladas.

Con el fin anterior en este trabajo se modela y simula, con Simulink ${ }^{\circledR}$ de Matlab®, un aerogenerador de baja potencia, en este caso de $50 \mathrm{~kW}$, con tecnología de conversión de la energía eólica (WECS) para generador sincrónico a imanes permanentes (PMSG) de polos no salientes, acoplamiento directo (Gear Less), de velocidad variable y paso de pala. Utiliza un esquema de control para captura de máxima energía del viento (MPPT, en zona de carga parcial), que aplica al PMSG un seteo de la corriente directa estatórica a cero amperes (control ZDC).

Por otra parte emplea una tecnología de conversión (back to back) en dos niveles, con modulación vectorial, en donde la barra de continua (DC) se respalda a través de un banco de baterías, con capacidad dependiente del estudio de la carga, y recurso eólico del sitio.

Se presentan algunas de las salidas principales de las simulaciones, para una determinada condición del recurso, cuyo análisis de desempeño justifican el desarrollo de un futuro prototipo que las valide.

Palabras clave: Eólica, Conversión, Control, Vectorial. 


\section{Abstract}

There is a today given technical and economic aspect, feasibility of supplying electricity through wind generation, where the resource permits, small isolated communities.

With the above purpose in this work is models and simulates with Simulink ${ }^{\circledR}$ of Matlab ${ }^{\circledR}$ a wind turbine low power, in this case of $50 \mathrm{~kW}$, powered by wind energy conversion (WECS) for permanent magnet synchronous generator (PMSG ) no pole outgoing, direct coupling (Less Gear), variable speed and blade pitch. Uses a control scheme to capture maximum wind energy (MPPT in partial load), which applies to a config PMSG direct stator current to zero amps (ZDC control).

Moreover employs a conversion technology (back to back) on two levels, with vector modulation, where the bar (DC) is supported by a battery bank with capacity dependent study of the load, and wind resource site.

They are presented some of the main outputs of the simulations, for a given resource condition, the performance analysis justify the development of a prototype that validates future.

Key words: Wind, Conversion, Control, Vector. 


\section{Introducción}

El sistema de conversión aplicado (PMSG) surge de los desarrollos en generación eólica en grandes potencias como lo indican las tendencias (Ackermann, 2005). Utiliza un sistema de conversión completa como el indicado en la Figura 1 de manera de desvincular generación y frecuencia variable (Teodorescu, 2011; $\mathrm{Wu}, 2011$ ), alimentando a una demanda con características del producto técnico en valores dentro de límites impuestos por las recomendaciones y normas (Estanqueiro, 2007; IEC 61400-21, 2001; IEC 61000-4-7, 2002).

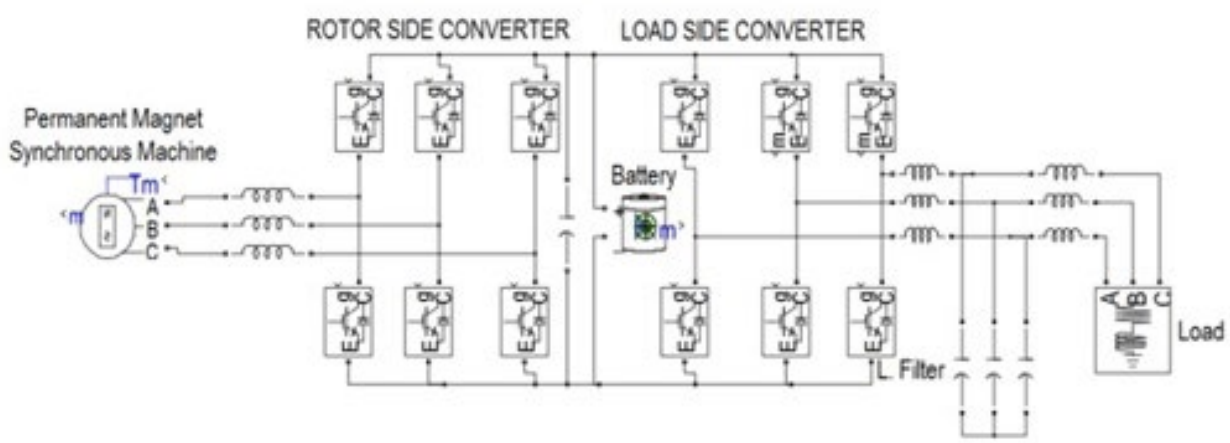

Figura 1 diagrama simplificado, full-converter con tecnología espalda con espalda (back to back), empleado en el modelo.

Dentro del esquema de conversión de potencia el rotor side converter cumple la función de maximizar captura de energía del viento por parte del rotor, controlando flujo de potencia hacia el lado carga y velocidad de giro óptima. En la Figura 2 se muestra el concepto del esquema MPPT, en donde se observa una familia de curvas de potencia aerodinámicas (función del viento incidente) y el lugar de trabajo que el control ubica a la curva de potencia mecánica transmitida, la cual es proporcional al cubo de la velocidad de rotación.

Superada la velocidad de viento a la que se obtiene la potencia nominal, actúa el control de paso de pala de manera de disminuir el coeficiente de potencia $(\mathrm{CP}=\mathrm{f}$ $(\lambda, \beta))$, en donde " $\lambda$ " es la relación de velocidad en punta de pala y " $\beta$ " el paso de pala (Burton, 2001).

$$
\mathrm{Pm}=1 / 2 * \mathrm{CP} * \rho^{*} \mathrm{~A} * \mathrm{~V}_{\infty}^{3}
$$

Pm $=$ Potencia mecánica de trabajo $\rho=$ Densidad del aire

$\mathrm{CP}=$ Coeficiente de potencia $\mathrm{V} \infty \mathrm{V}=$ Velocidad del viento

$\mathrm{A}=$ área barrida por el rotor del aerogenerador 
El generador PMSG puede ser controlado por diversos métodos (Fox, 2007; Bianchi, 2007; Munteanu, 2008), se opta en este trabajo dado su buen desempeño y facilidad de modelado por el ZDC (Wu, 2011), donde las tres corrientes de fase estatórica, en el marco de referencia estacionario, son transformadas en uno de referencia sincrónico rotante a la pulsación de giro de la máquina sincrónica multiplicada por el número de pares de polos. De esta manera se logra pasar de tres variables de alterna a dos de continua y facilitar la tarea de control buscada. Estas corrientes se encuentran en cuadratura representadas por la componente en directa "d" y cuadratura "q", (Anaya-Lara, 2009). El control setea la componente del eje "d" a cero de allí el nombre del control (Zero d-axis Current) de manera de llevar una proporcionalidad entre la corriente estatórica y el torque electromagnético (Te).

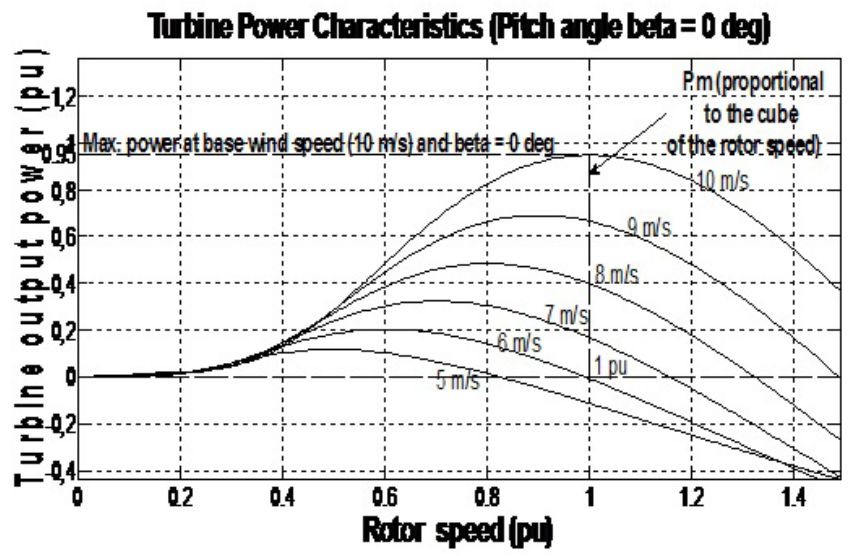

Figura 2 Curvas de potencia aerodinámica versus potencia mecánica de trabajo en función de velocidad de viento. Eje de ordenadas en unidades ponderadas respecto a la potencia nominal de la turbina.

$$
\overrightarrow{\mathrm{I}}_{\mathrm{s}}=\mathrm{i}_{\mathrm{ds}}+\mathrm{ji}_{\mathrm{qs}}=\mathrm{ji}_{\mathrm{qs}} \quad \text { para } \mathrm{i}_{\mathrm{ds}}=0
$$

Por otro lado de acuerdo a (Krause, 2002), el torque electromagnético desarrollado por el generador en cuestión responde a

$$
\mathrm{Te}=3 / 2 * \mathrm{P} *\left(\phi_{\mathrm{r}} * \mathrm{i}_{\mathrm{qs}}-\left(\mathrm{L}_{\mathrm{d}}-\mathrm{L}_{\mathrm{q}}\right) * \mathrm{i}_{\mathrm{qs}} * \mathrm{i}_{\mathrm{ds}}\right)
$$

$\mathrm{Te}=$ Torque electromagnético

$\mathrm{P}=\mathrm{N}^{\circ}$ de pares de polos de la máquina sincrónica

$\Phi \mathrm{r}=$ Flujo de enlace producido por lo imanes permanentes

$\mathrm{Ld}$ y $\mathrm{Lq}=$ Inductancias estatóricas en el marco "d q"

$\mathrm{i}_{\mathrm{ds}}=$ Amplitud de corriente directa estatórica en el marco "d q"

$\mathrm{i}_{\mathrm{qs}}=$ Amplitud de corriente en cuadratura estatórica en el marco "d q" 
Ya que para una máquina sincrónica de polos no salientes se cumple que $\mathrm{Ld}=$ Lq, (Wu, 2011), con lo cual

$$
\mathrm{Te}=3 / 2 * \mathrm{P} * \phi_{\mathrm{r}} * \mathrm{i}_{\mathrm{qs}}
$$

Lo último, como se indicó, exhibe una relación lineal entre el torque y la corriente estatórica, lo cual asemeja el comportamiento a una máquina de DC de excitación independiente, donde el torque es proporcional a la corriente de armadura.

Por el lado de las tensiones desarrolladas en el marco rotante sincrónico, con este esquema, se tiene

$$
\begin{aligned}
& \overrightarrow{\mathrm{v}}_{\mathrm{s}}=\mathrm{v}_{\mathrm{ds}}+\mathrm{jv}_{\mathrm{qs}}=\omega_{\mathrm{s}} * \mathrm{~L}_{\mathrm{q}} * \mathrm{i}_{\mathrm{qs}}+\mathrm{j} \omega_{\mathrm{s}} * \phi_{\mathrm{r}} \\
& \omega_{\mathrm{s}}=\text { pulsación sincrónica del estator }
\end{aligned}
$$

Por lo tanto podemos inferir el ángulo del factor de potencia estatórico

$$
\varphi_{\mathrm{s}}=\varphi_{\mathrm{v}}-\varphi_{\mathrm{i}}
$$

Con

$$
\begin{aligned}
& \varphi_{\mathrm{v}}=\tan ^{-1} \mathrm{v}_{\mathrm{qs}} / \mathrm{v}_{\mathrm{ds}} \\
& \varphi_{\mathrm{i}}=\tan ^{-1} \mathrm{i}_{\mathrm{qs}} / \mathrm{i}_{\mathrm{ds}}
\end{aligned}
$$

Ya que $\mathrm{i}_{\mathrm{ds}}=0 \quad \varphi_{\mathrm{i}}=\pi / 2$

Por otro lado, partiendo del concepto que para el control de la conversión de la energía eólica se cumple (Pao, 2009).

$\dot{\omega}_{\mathrm{r}}=1 / \mathrm{J} *\left(\mathrm{~T}_{\mathrm{m}}-\mathrm{T}_{\mathrm{e}}\right)$

$\grave{\omega}_{\mathrm{r}}=$ Aceleración angular rotórica

$\mathrm{T}_{\mathrm{m}}=$ Torque mecánico

$\mathrm{J}=$ Momento de inercia generador-rotor

El sistema, a través del control, compensará dicha variación de torque manteniendo la aceleración angular en su valor óptimo. Teniendo en cuenta que el torque mecánico, en estado estacionario, corresponde a 


$$
\begin{aligned}
& \mathrm{T}_{\mathrm{m}}=1 / 2 * \mathrm{CP} * \rho * \pi * \mathrm{R}^{5 *} \omega^{2} / \lambda^{3} \\
& \mathrm{R}=\text { Radio de pala }
\end{aligned}
$$

Igualando $\mathrm{T}_{\mathrm{m}}$ y $\mathrm{T}_{\mathrm{e}}$, con las consideraciones del caso, queda que la corriente de referencia a aplicar al controlador es proporcional a la velocidad angular rotórica al cuadrado.

$$
\mathrm{i}_{\mathrm{qs}}^{*}=\frac{\mathrm{CP}_{\mathrm{opt}} * \rho * \pi * \mathrm{R}^{5}}{3 * \mathrm{P} * \phi_{\mathrm{r}} * \lambda_{\mathrm{opt}}{ }^{3}} * \omega_{\mathrm{r}}{ }^{2}
$$

$\mathrm{CP}_{\text {opt }}=$ Coeficiente de potencia máximo u óptimo

$\lambda_{\mathrm{opt}}=$ Relación de punta de pala óptima

$\mathrm{i}_{\mathrm{qS}}{ }_{\mathrm{os}}=$ Corriente de referencia a aplicar al controlador

Ya que como se indicó se trabaja con un control ZDC, se fija

$$
\mathrm{i}_{\mathrm{qS}}^{*}=0
$$

Ambas corrientes de referencia se aplican al controlador formado por PI (proporcional integral) de cuyas salidas se obtienen las tensiones de referencia en el marco sincrónico rotante, estas se transforman nuevamente al marco estacionario de tres variables, obteniéndose las señales de referencia a aplicar al convertidor lado rotor y producir el disparo de los interruptores de potencia según la referencia buscada.

En la siguiente Figura 3 se muestra un modelo simplificado del esquema de control empleado.

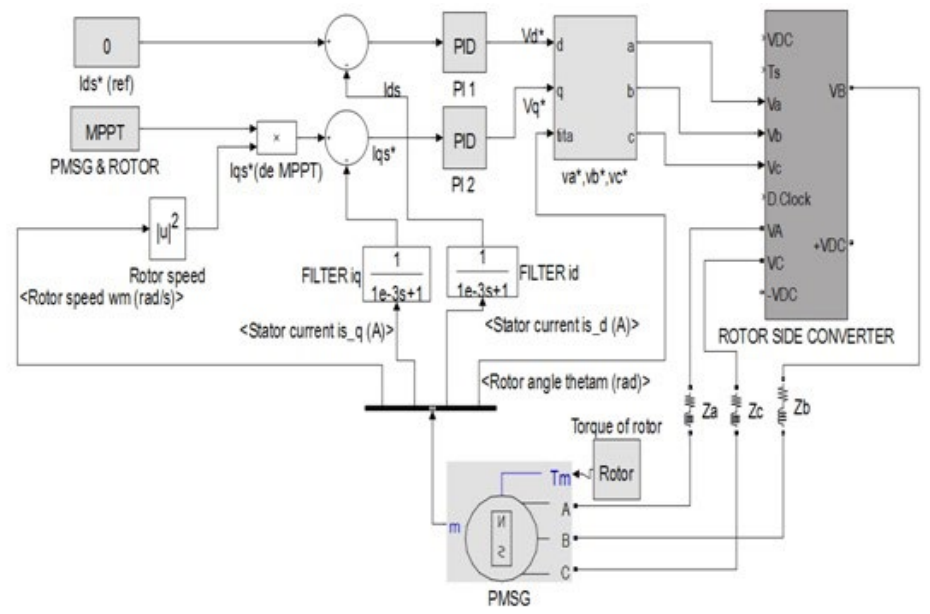

Figura 3 Control ZDC para conversión de energía eólica "WECS" 


\section{Conversión espalada con espalda, de dos niveles, en modulación vectorial}

El flujo de potencia es conducido a través de un arreglo de conversión espalda con espalda, en donde dos convertidores con un enlace de corriente continua, en este caso soportado por un banco de baterías, suministran la potencia a una carga de corriente alternada.

Se aplica para la conmutación de los interruptores de potencia una técnica de modulación vectorial "SVM", (Wang, 2010; Wu, 2011) permitiendo conversiones de bajo contenido armónico lo cual contribuye a menor desempeño en filtrados especiales, reduciendo costo, espacio y pérdida de potencia.

La conversión de corriente continua a alterna y viceversa, se logra a través de diferentes técnicas de modulación y según estas con diversos arreglos (Dias Filho, 2009; Yazdami, 2010).

Para lo anterior, partiendo del siguiente esquema de Figura 4 de conversión simplificado de dos niveles, se definen según Tabla 1 los estados posibles de los interruptores de potencia.

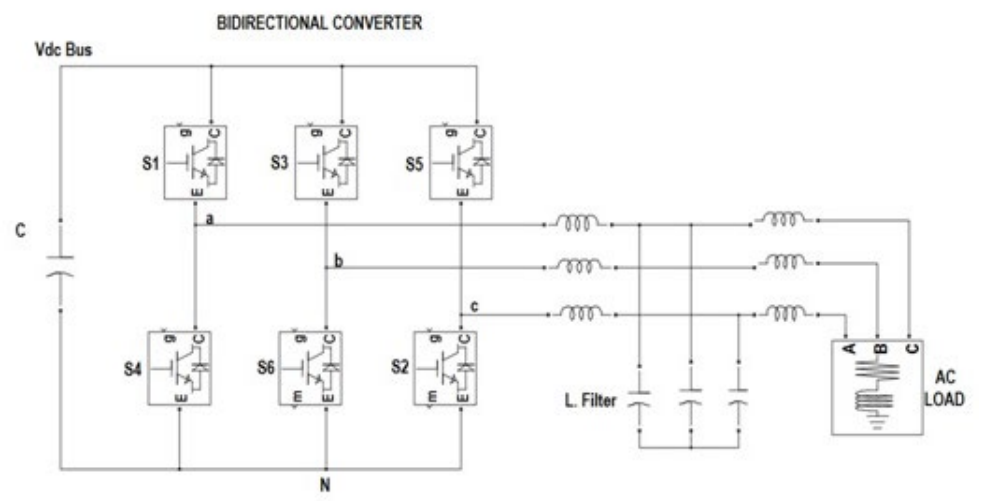

Figura 4 Convertidor bidireccional simplificado de dos niveles

Tabla 1 Definición del estado de los interruptores

\begin{tabular}{|c|c|c|c|c|c|c|c|c|c|}
\hline \multirow[b]{2}{*}{ Est. Interruptores } & \multicolumn{3}{|c|}{ Rama a } & \multicolumn{3}{|c|}{ Rama b } & \multicolumn{3}{|c|}{ Rama c } \\
\hline & S1 & $\mathrm{S} 4$ & $\mathrm{VaN}$ & S3 & S6 & $\mathrm{VbN}$ & S5 & S2 & $\mathrm{VcN}$ \\
\hline 1 & On & Off & $\mathrm{Vdc}$ & On & Off & $\mathrm{Vdc}$ & On & Off & $\mathrm{Vdc}$ \\
\hline 0 & Off & On & 0 & Off & On & 0 & Off & On & 0 \\
\hline
\end{tabular}


La conversión en dos niveles permite seis posibles estados activos representados por vectores estacionarios según la siguiente Figura 5 y Tabla 2.

La idea consiste en reconstruir en potencia un vector rotante de referencia, el cual representa una composición de señales trifásicas, que pueden ser transformadas en cada instante en un marco estacionario de dos variables $(\alpha \beta)$ (Wu, 2011), cuyo giro coincide con la pulsación o frecuencia de la señal trifásica a conformar en potencia sobre la carga.

En Tabla 2 se desarrolla el espacio vectorial estacionario utilizado para sintetizar por cada muestra "Ts", en tiempo real, los estados y tiempos de conmutación de los interruptores de potencia.

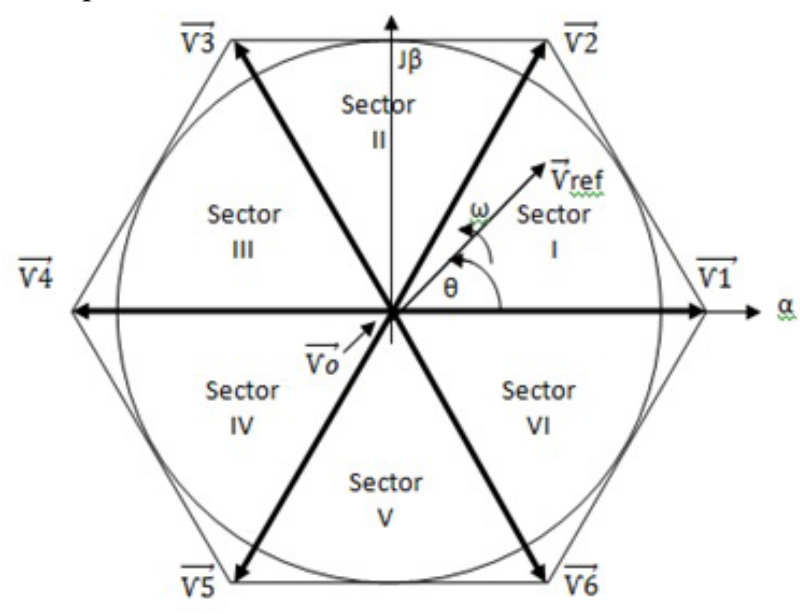

Figura 5 Espacio vectorial convertidor dos niveles

El vector de referencia es sintetizado en cada muestra, según el sector en que se encuentra, por dos vectores activos adyacentes a su ubicación temporal y los "nulos" que permiten reducir, entre otras, la frecuencia de conmutación de los interruptores de potencia.

Por lo tanto, partiendo de una referencia balanceada

$$
V a(t)+V b(t)+V c(t)=0
$$

Transformamos al marco de referencia estacionario como

$$
\begin{aligned}
& \vec{V}(t)=V \alpha(t)+j V \beta(t) \\
& \vec{V}(t)=\frac{2}{3}\left(V a(t) e^{j 0}+V b(t) e^{\frac{j 2 \pi}{3}}+V c(t) e^{\frac{j 4 \pi}{3}}\right)
\end{aligned}
$$


Los estados y amplitudes de Tabla 2 coinciden con el instante del vector de referencia en dichas ubicaciones. Llamando " $k$ " a los seis sectores enmarcados por los vectores estacionarios, podemos escribir

$$
\vec{V}(t=t x)=\overrightarrow{V r e f}=\operatorname{Vref} e^{j \theta}=\frac{2}{3} \operatorname{Vdc} e^{j(k-1) \pi / 3}
$$

Cada muestra de tiempo "Ts", es dividida en siete tiempos denominados de permanencia de estados de conmutación ( $\mathrm{Wu}, 2011)$. Este número particular permite minimizar la frecuencia de conmutación de los interruptores, recordar que las pérdidas de potencia de los mismos es función directa de dicho valor.

Ya que el vector rotante se sintetiza por tres vectores, dos activos (con magnitud no nula) y un tercero nulo ( $\mathrm{Wu}, 2011)$, el tiempo Ts quedará dividido en cada muestra como sigue

$$
\mathrm{Ts}=\mathrm{Ta}+\mathrm{Tb}+\mathrm{To}
$$

\begin{tabular}{|c|c|c|c|}
\hline Espacio vectorial & Definición vectorial & estado de conmutación & intemuptores en ON \\
\hline \multicolumn{4}{|l|}{ Nulo o Cero } \\
\hline $\overrightarrow{V_{0}}$ & $\overrightarrow{V_{0}}=0$ & 111,000 & $\mathrm{~S} 1, \mathrm{~S} 3, \mathrm{~S} 5$ y S4, S6, S2 \\
\hline \multicolumn{4}{|l|}{ Activo } \\
\hline $\overrightarrow{\mathrm{V} 1}$ & $\overrightarrow{V 1}=\frac{2}{3} V d c \cdot e^{j 0}$ & 100 & $\mathrm{~S} 1, \mathrm{~S} 6, \mathrm{~S} 2$ \\
\hline $\overrightarrow{\mathrm{V} 2}$ & $\overrightarrow{V 2}=\frac{2}{3} V d c \cdot e^{j \pi / 3}$ & 110 & $\mathrm{~S} 1, \mathrm{~S} 3, \mathrm{~S} 2$ \\
\hline $\overrightarrow{\mathrm{V} 3}$ & $\overrightarrow{\mathrm{V} 3}=\frac{2}{3} \mathrm{Vdc} \cdot \mathrm{e}^{\mathrm{j} 2 \pi / 3}$ & 010 & $\mathrm{~S} 4, \mathrm{~S} 3, \mathrm{~S} 2$ \\
\hline $\overrightarrow{\mathrm{V} 4}$ & $\overrightarrow{\mathrm{V} 4}=\frac{2}{3} \mathrm{Vdc} \cdot \mathrm{e}^{\mathrm{j} \pi / 3}$ & 011 & S4, S3, S5 \\
\hline $\overrightarrow{\mathrm{V} 5}$ & $\overrightarrow{V 5}=\frac{2}{3}$ Vdc. $\mathrm{e}^{\mathrm{j} 4 \pi / 3}$ & 001 & S4, S6, S5 \\
\hline $\overrightarrow{\mathrm{v} 6}$ & $\overrightarrow{V 6}=\frac{2}{3} V d c \cdot \mathrm{e}^{\mathrm{j} 5 \pi / 3}$ & 101 & S1, S6, S5 \\
\hline
\end{tabular}

Tabla 2 Espacio vectorial y estados de conmutación de los vectores estacionarios.

"Ta" "Tb" y "To" son los tiempos de permanencia, dentro de cada muestra Ts, de los estados de conmutación según los vectores activos y nulos en que se enmarque el vector de referencia en tiempo real en cada instante. Surgen de un balance voltssegundos entre vector de referencia y tiempo de muestra, con vectores en que se sintetiza y sus tiempos de permanencia.

Se obtienen matemáticamente haciendo las operaciones con datos de Tabla 3

$$
\begin{aligned}
& \mathrm{Ta}=\frac{\sqrt{3} \text { Vref Ts }}{\operatorname{Vdc}} \operatorname{sen}\left(\begin{array}{ll}
\pi & \theta^{\prime} \\
3 &
\end{array}\right) \\
& \mathrm{Tb}=\frac{\sqrt{3} \text { Vref Ts }}{\operatorname{Vdc} \operatorname{sen} \theta^{\prime}}
\end{aligned}
$$


Para $\quad \theta^{\prime}-\theta-(k-1) \frac{\pi}{3} \quad 0<6^{\prime}<\frac{\pi}{3}$

$\mathrm{k}=$ número de sector

De esta manera se puede calcular para cada muestra Ts, según la ubicación del vector de referencia (el sector en que se encuentre), el tiempo de permanencia de cada estado de conmutación de los interruptores del convertidor de potencia.

Concluido un giro completo (a la pulsación elegida), se vuelve a repetir un nuevo ciclo de reconstrucción en potencia de la referencia.

Esta modulación en el espacio vectorial posee un índice, al igual que la SPWM, denominado "ma" que define la amplitud de la componente fundamental a obtener a la salida del convertidor.

$\mathrm{ma}=\frac{\sqrt{ } 3}{\mathrm{Vdc}} \mathrm{Vref}$

Procediendo matemáticamente se obtiene

$$
\mathrm{Ta}=\mathrm{ma} \operatorname{Ts} \operatorname{sen}\left(\begin{array}{ll}
\pi & \theta^{\prime} \\
3 &
\end{array}\right) \quad \mathrm{Tb}=\mathrm{ma} \mathrm{Ts} \operatorname{sen} \theta^{\prime}
$$

El método planteado no permite sobremodulación ( $\mathrm{ma}>1$ ), mismo el vector de referencia debe quedar, para evitar cálculos de tiempos permanencia no validos, inscripto dentro del círculo mostrado en la Figura 5.

Establecidas las pautas anteriores se plantea, a través de la Tabla 3, un arreglo de los tiempos de permanencia de los estados de conmutación, en donde cada muestra se divide a su vez en siete segmentos de estados de conmutación. Según sea el sector par o impar donde se encuentre el vector de referencia, se realiza y modela un intercambio entre los tiempos de permanencia correspondiente a los vectores activos adyacentes a dicha referencia. El arreglo intenta y logra que en cada muestra solo haya un cambio de encendido a apagado y viceversa por cada interruptor, mismo que al pasar de un estado de conmutación al contiguo solo haya cambio en una sola rama del convertidor, minimizando de esta manera la frecuencia de conmutación de dichos interruptores de potencia.

Esto permite ser coherente con el desarrollo analítico, y conforme el arreglo, por medio en principio de modelado y simulación mejorar la distorsión armónica total (THD). 
Tabla 3 Arreglo de estados y tiempos de conmutación propuesto

\begin{tabular}{|c|c|c|c|c|c|c|c|}
\hline & $\mathrm{To} / 4$ & $\mathrm{~Tb} / 2$ & $\mathrm{Ta} / 2$ & $\mathrm{To} / 2$ & $\mathrm{Ta} / 2$ & $\mathrm{~Tb} / 2$ & $\mathrm{To} / 4$ \\
\hline Sector I & 111 & 110 & 100 & 000 & 100 & 110 & 111 \\
\hline Sector III & 111 & 011 & 010 & 000 & 010 & 011 & 111 \\
\hline \multirow[t]{3}{*}{ Sector V } & 111 & 101 & 001 & 000 & 001 & 101 & 111 \\
\hline & & & & Ts & & & \\
\hline & $\mathrm{To} / 4$ & $\mathrm{Ta} / 2$ & $\mathrm{~Tb} / 2$ & $\mathrm{To} / 2$ & $\mathrm{~Tb} / 2$ & $\mathrm{Ta} / 2$ & $\mathrm{To} / 4$ \\
\hline Sector II & 111 & 110 & 010 & 000 & 010 & 110 & 111 \\
\hline Sector IV & 111 & 011 & 001 & 000 & 001 & 011 & 111 \\
\hline Sector VI & 111 & 101 & 100 & 000 & 100 & 101 & 111 \\
\hline
\end{tabular}

En base a lo expuesto, y mostrado en Figura 1, se modelan dos convertidores iguales según el siguiente esquema de bloques de Figura 6, (Wu, 2011).

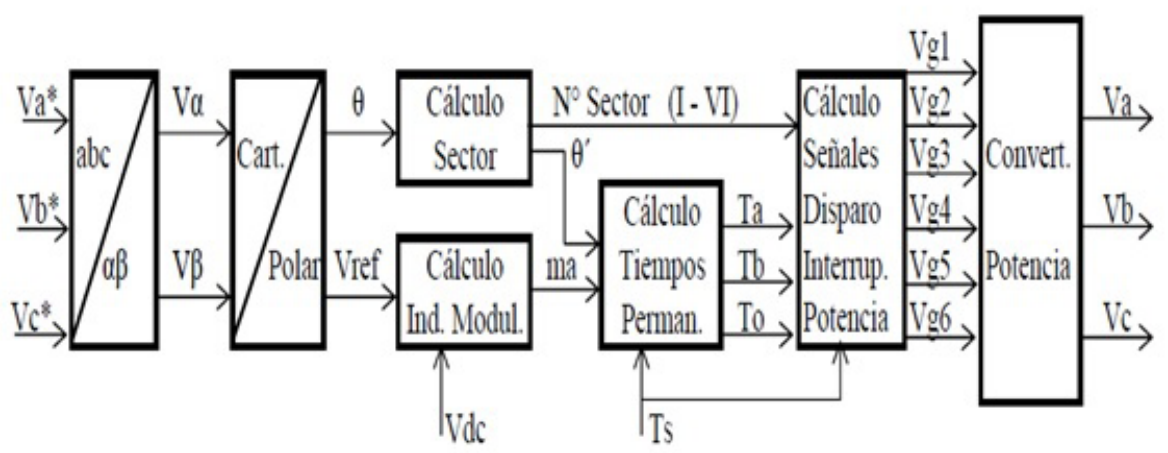

Figura 6 Diagramas de bloques de modelado y simulación SVM para conversión de dos niveles 


\section{Esquema final de modelado de conversión de energía eólica pmsg}

En la siguiente Figura 7 se presenta el modelo final, según objetivo del trabajo, de un "WECS" completo cuyas características principales son las siguientes:

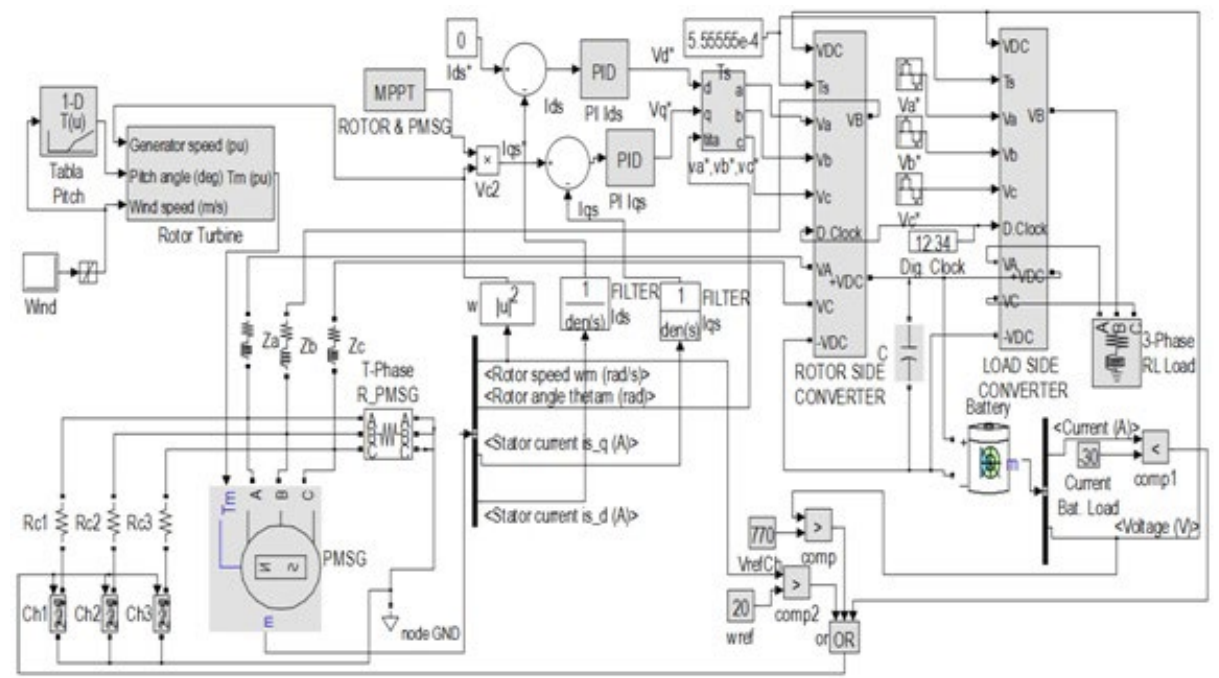

Figura 7 Esquema de modelo "WECS" PMSG con control ZDC

- Modelo de rotor en potencia mecánica nominal de $51.500 \mathrm{~kW}$, paso de pala óptimo en $\beta=0^{\circ}$ y velocidad de viento nominal $\mathrm{V}_{\infty}=10.27 \mathrm{~m} / \mathrm{s}$, velocidad de rotación nominal $\omega=10.3 \mathrm{r} / \mathrm{s}$ con radio de pala estimado en $\mathrm{R}=7,17 \mathrm{~m}$, torque Figura 7 Esquema de modelo "WECS" PMSG con control ZDC

- Modelo de rotor en potencia mecánica nominal de $51.500 \mathrm{~kW}$, paso de pala óptimo en $\beta=0^{\circ}$ y velocidad de viento nominal $\mathrm{V} \infty=10.27 \mathrm{~m} / \mathrm{s}$, velocidad de rotación nominal $\omega=10.3 \mathrm{r} / \mathrm{s}$ con radio de pala estimado en $\mathrm{R}=7,17 \mathrm{~m}$, torque mecánico nominal $\mathrm{Tm}=5000 \mathrm{Nm}$. Estimando un momento de inercia según (González Rodríguez, 2013) de alrededor de J $=2100$ kg*m2.

- Modelo de generador sincrónico a imanes permanentes PMSG de potencia nominal $\mathrm{Pg}=51.500 / 0.9 \mathrm{VA}$, polos no salientes $\mathrm{P}=12$ (pares de polos), Inductancias en cuadratura $\mathrm{Ld}=\mathrm{Lq}=5^{\star} 10^{-3} \mathrm{Hy}$, flujo establecido por los imanes $\phi \mathrm{r}=3 \mathrm{~V} . \mathrm{s}$ y constante de tensión de salida $\mathrm{Kv}=6530 \mathrm{~V}$

$\widehat{\mathrm{V}} \mathrm{L}-\mathrm{L} / \mathrm{krpm}$

- Modelado de MPPT en base a datos de rotor y generador sincrónico PMSG, con variación proporcional del torque a la velocidad de giro al cuadrado.

- Modelado del control ZDC de acuerdo a (Wu, 2011) y sintonía de controladores PI según regla de sintonía de Ziegler-Nichols (Ogata, 2010) esto último dado lo complejo de la planta y el desconocimiento de su exacta función de transferencia. 
- Modelado de convertidores de potencia con interruptores IGBT, índice de modulación nominal ma $=0.8$, tensión de alimentación de carga $\mathrm{V}$ L-L $=380$ $\mathrm{V}$, frecuencia de muestro $\mathrm{fs}=1800 \mathrm{~Hz}, \mathrm{y}$ carga con ángulo de factor de potencia $\mathrm{PF}=0.9$.

- Modelado de banco de acumuladores, (sin análisis del recurso eólico particular del sitio y necesidades de autonomía), ejemplo compuesto de 54 baterías de 12 $\mathrm{V}$ (648 V nominal) y $150 \mathrm{AH}$ de capacidad (una hora aproximada de autonomía a plena carga, $50 \mathrm{~kW})$.

- Modelado de "Dump Loads", a través de circuitos de Chopper, para evitar sobre carga de banco de acumuladores y control de emergencia de velocidad de giro.

\section{Desempeño De Modelo (WECS) PMSG}

En las siguientes figuras se muestran las salidas de simulación principales del modelo propuesto.

En Figura 8 de más arriba se puede observar el buen desempeño del modelo, en donde al aplicar un viento incidente de valor indicado, con una crecida (rising slew rate $=10$ de manera de emular un aumento de viento real), pasado un tiempo (dado la cte. de inercia del rotor más generador) se estabiliza la velocidad de giro en coincidencia con el encuentro entre el torque mecánico y electromagnético, lo cual valida lo planteado matemáticamente en la introducción de este trabajo.

La Figura 9 permite observar la entrega de una corriente de fase del generador PMSG de alrededor de los 82 A, lo cual valida el control ZDC y a través de este él MPPT. Ya que reemplazando dicho valor en expresión planteada del torque electromagnético de párrafos de introducción, para el generador PMSG de polos no salientes, coincide dicho resultado con el valor final mostrado en Figura 8 de los torques en alrededor de los $4400 \mathrm{Nm}(0.88 \mathrm{pu})$, con una velocidad de rotación de casi 1 pu (cercana a los $10 \mathrm{r} / \mathrm{s}$ ).

Por otro lado, más allá de la deformación de la tensión, hay una coincidencia en fase entre ambas señales, infiriendo un factor de potencia hacia el generador muy cercano a la unidad.

En la Figura 10 se observa la corriente de batería (valores positivos cuando entrega corriente y negativos cuando recibe). El sistema aparte de alimentar la carga ( $40 \mathrm{~kW}, \mathrm{FP}=0.9)$, comienza a cargar la batería en determinados instantes, necesitando para este caso tan solo una media de batería no mayor a los $5 \mathrm{~A}$. 


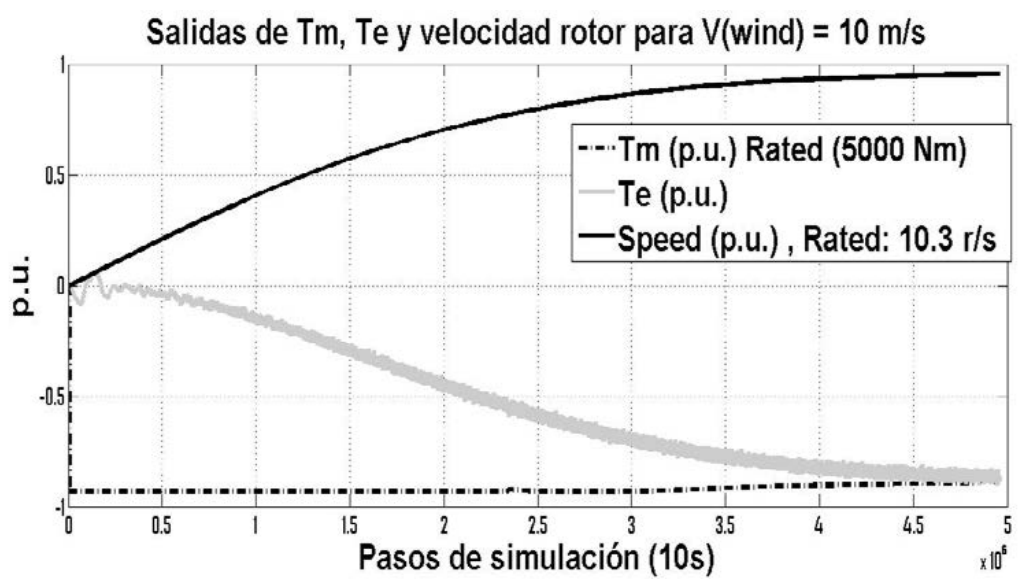

Figura $8 \mathrm{Tm}$, Te y velocidad de rotor, para V (Wind) $=10 \mathrm{~m} / \mathrm{s}$

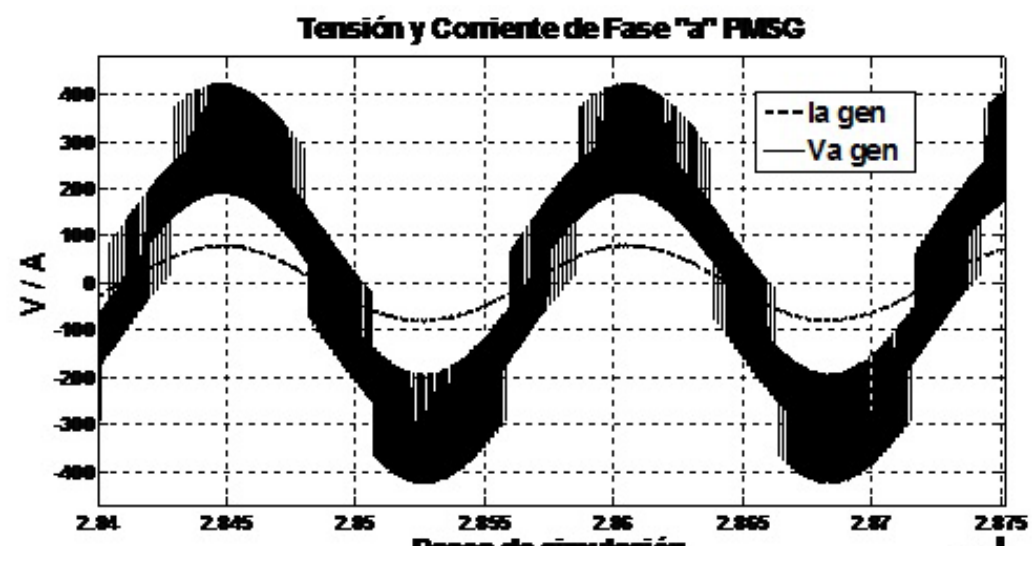

Figura 9 Tensión y corriente de Fase "a" PMSG

A medida pasa el transitorio, para el viento planteado, se llega a velocidad de rotación cercana a $1 \mathrm{p}$.u, dando una potencia entregada de alrededor de los $48 \mathrm{~kW}$ con pérdidas internas del orden de los $4 \mathrm{~kW}$ (rendimiento mayor al 90\%), permitiendo la carga de batería con el exceso de potencia generada.

La Figura 11 entrega las corrientes en el marco sincrónico "d q" que surgen de la transformación de las medidas de corriente en el generador PMSG del marco estacionario trifásico. Dichos valores finales de corriente, corroborado con lo obtenido en curva de corriente de fase de Figura 9, ratifican el buen desempeño del control ZDC.

Por último la Figura 12 muestra la corriente de referencia "Iqs*" que surge de datos del PMSG y rotor que permiten el MPPT, observándose una coincidencia o seguimiento de la misma por parte del controlador, lo cual se evidencia al comparar con la de Figura 11. 


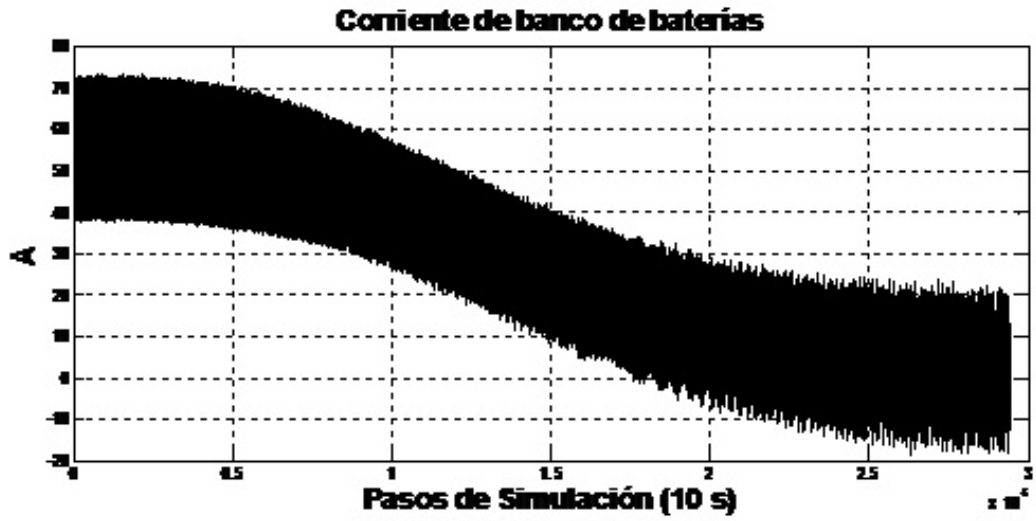

Figura 10 Corriente de banco de baterías

Corriente en marco sincrónico "d q" medidas en PMSG

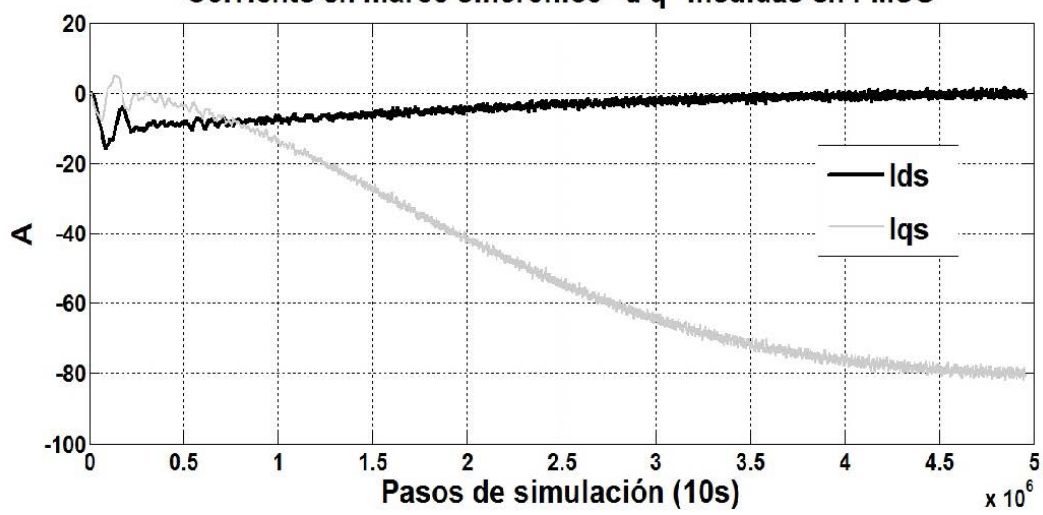

Figura 11 Corrientes medidas de PMSG en marco sincrónico

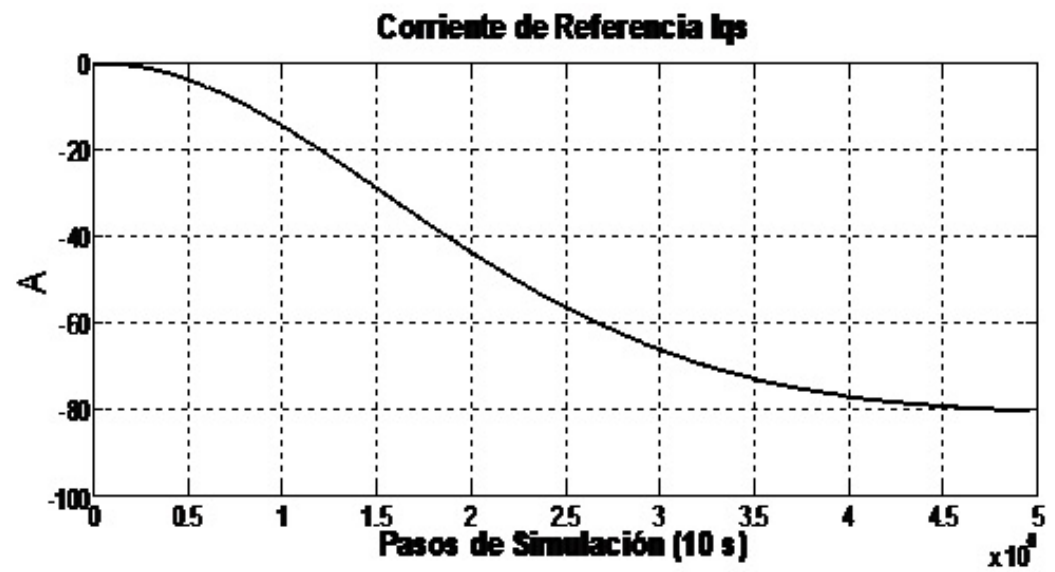

Figura 12 Corrientes de referencia Iqs* obtenida de MPPT 


\section{Conclusiones}

El modelo, en etapa de simulación, entrega índices que validan la bondad de la propuesta desarrollada. Permite, en principio dado los costos, realizar pruebas de desempeño a través de prototipo en potencia menor a la planteada (del orden del $\mathrm{kW}$ ).

Una mayor frecuencia de muestreo permitirá mejor desempeño global del sistema $(10 \mathrm{kHz})$. Se trabaja a menor valor $(1.8 \mathrm{kHz})$ con el fin de disminuir el consumo de recurso informático y por ende reducir los tiempos de las corridas. Dicho valor de muestreo sin embargo es indicador del buen funcionamiento del modelo elaborado.

Aplica y verifica lo hecho en trabajo de tesis de maestría (Bufanio, R. 2013) donde se modela y simula una propuesta de convertidor en dos niveles en modulación SVM con arreglo de disparo de los interruptores de potencia que brinda resultados satisfactorios de THD a la salida del convertidor.

Corrobora el buen desempeño del control de potencia tanto en carga parcial como total, en donde en la primera (por debajo del viento nominal) el MPPT permite adquirir la máxima potencia del viento incidente. Superado dicho límite el control de paso de pala actúa correctamente de manera de mantener la potencia a la nominal. 


\section{Referencias}

Ackermann, T. (2005). Wind Power in Power Systems. London: John Wiley and Sons, Ltd.

Anaya-Lara, O., Jenkins, N., Ekanayake, J., Cartwright, P. \& Hughes, M. (2009). Wind Energy Generation, Modeling and Control. London: John Wiley and Sons, Ltd.

Bianchi, F., Battista H. \& Mantz, R. (2007). Wind Turbine Control Systems. London: Springer-Verlag London Limited.

Bufanio, R. (2013). Recomendación y Propuesta para la Optimización del Contenido Armónico en la Generación Eólica PMSG. Universidad Tecnológica Nacional, Facultad Regional Córdoba, Argentina.

Burton, T., Sharpe, D., Jenkins, N. \& Bossanyi, E. (2001). Wind Energy Handbook. London: John Wiley and Sons, Ltd.

Dias Filho, R. (2009). Análisis de Técnicas de Control de Conversores Conectados a Redes Eléctricas. Universidad Federal de Pernambuco, Recife, Brasil.

Estanqueiro, A. \& Tande, J. (2007, June). Assessment of Power Quality Characteristics of Wind Farm. Power Engineering Society General Meeting, Tampa Florida, USA.

Fox, B., Flynn, D., Bryans, L., Jenkins, N., Milborrow, D., O’Malley, M., Watson, R. \& Anaya-Lara, O. (2007). Wind Power Integration. London: Institution of Engineering and Technology.

Gonzalez Rodriguez, G., Gonzalez Rodriguez, A. \& Burgos Payán, M. (2013). Estimating Wind Turbines Mechanical Constants. España: University of Jaén, of Castilla La Mancha and of Seville, Betty Blue.

International Electrotechnical Commission. (2001). IEC 61400-21, Wind Turbine Generator Systems, Part 21: Measurement and Assessment of Power Quality Characteristics of Grid Connected Wind Turbines.

International Electrotechnical Comisison. (2002). IEC 61000-4-7, Electromagnetic Compatibility EMC, Parts 4-7: Testing and Measurements Techniques. General Guide on Harmonics and Interharmonics Measurements and Instrumentation, for Power Supply Systems and Equipment Connected.

Krause, P., Wasinczuk, O. \& Sudhoff, S. (2002). Analysis of Electric Machinery and Drive Systems (2da Ed.). New York: John Wiley and Sons, Inc. 
Munteanu, I., Bratcu, A., Cutululis., N. \& Seanga, E. (2008). Optimal Control of Wind Power Systems. London: Springer-Verlag London Limited.

Ogata, K. (2010). Ingeniería de Control Moderna (5ta Ed.). Madrid: Pearson Educación, S.A.

Pao, L. \& Johnson K. (2009). A Tutorial on the Dynamics and Control of Wind Turbines and Wind Farms. American Control Conference, St. Louis, USA.

Teodorescu, R., Liserre, M. \& Rodriguez, P. (2011). Grid Converters for Photovoltaic and Wind Power Systems. London: John Wiley and Sons, Ltd.

Wang, L., Singh C. \& Kusiak, A. (2010). Wind Power Systems. London: SpringerVerlag London Limited.

Wu, B., Yongqiang, L., Zargari, N. \& Kouro, S. (2011). Power Convesion and Control of Wind Energy Systems. New Jersey: John Wiley and Sons, Inc.

Yazdami, A. \& Iravani, R. (2010). Voltage Sourced Converters in Power Systems. New Jersey: John Wiley and Sons, Inc. 